\title{
Endlich ein estnisches etymologisches Wörterbuch!
}

Iris Metsmägi, Meeli Sedrik, Sven-ErIK SoosaAR: Eesti etümoloogiasõnaraamat. [Estnisches etymologisches Wörterbuch.] Tallinn: Eesti Keele Sihtasutus, 2012. $792 \mathrm{~S}$.

Ein etymologisches Wörterbuch der estnischen Sprache ist seit Jahrzehnten ein Desideratum. Allerdings hatte Julius Mägiste in Schweden an einem solchen Wörterbuchprojekt gearbeitet, und nach seinem Tod wurde ein Facsimiledruck seines Manuskripts in den Jahren 1982-1983 von der FinnischUgrischen Gesellschaft veröffentlicht. Gleichzeitig, 1982, gab Alo Raun in Toronto ein etymologisches Wörterverzeichnis, Eesti keele etümoloogiline teatmik, heraus. Das erstere war in vielerlei Hinsicht unfertig und für das große Publikum in Estland weder zugänglich noch verwendbar, Rauns Büchlein stellte eigentlich nur eine minimalistisch kommentierte Wortliste dar. Ein echtes etymologisches Wörterbuch für die estnische Leserschaft gibt es also erst seit Mai 2012.

Dem neuen etymologischen Wörterbuch liegt natürlich eine längere Planungsarbeit zu Grun- de. Schon 1974 begannen die ForscherInnen des damaligen Instituts für Sprache und Literatur die vorhandene Information über die Geschichte der estnischen Wörter in einem Archiv zu sammeln; in dieser Kartothek gibt es momentan mehr als eine halbe Million Einträge. In der Anfangsphase wurde die Arbeit vor allem von Tiit-Rein Viitso und Paul Kokla geleitet. Diesen beiden, so wie auch dem nunmehr verstorbenen Karl Kont und Lembit Vaba, dem heutzutage wohl führenden Forscher über osfi.-baltische Sprachkontakte, wird im Vorwort ein besonderer Dank ausgedrückt.

Dennoch ist die Tradition der etymologischen oder überhaupt historisch-vergleichenden fiu. Sprachwissenschaft in Estland jünger oder zumindest dünner als in Finnland. Und dies nicht nur wegen der aus natürlichen Gründen knapperen Ressourcen, sondern auch, weil die estnische Finnougristik immer schon zu Zeiten F. J. Wiedemanns - eher synchron-deskriptiv als historisch orientiert war. Natürlich kann die Erforschung der estnischen Wortgeschichte in sehr vielen Fällen auf der finnischen Tradition der Etymologieforschung aufbauen. 
Dennoch bleiben der estnischen Etymologieforschung viele offene Fragen, vor allem auf dem Gebiet der jüngeren, spezifisch estnischen Lehnwortschichten. (Ganz davon $\mathrm{zu}$ schweigen, dass die finnischen EtymologInnen noch nicht immer unbedingt das letzte Wort zur Etymologie des osfi. Wortschatzes gesagt haben.)

Das Buch beginnt mit einer Einführung in die verschiedenen historischen Schichten des estnischen Wortschatzes, wohl größtenteils nach der oft zitierten Untersuchung von Huno Rätsep; von ihm wird in der Bibliografie nur die populäre Monografie Sõnaloo raamat (Buch der Wortgeschichte, 2002) erwähnt. Dabei wird implizit vom traditionellen „Binärbaum“ ausgegangen: die altererbten Wortstämme werden also in uralische, finnisch-ugrische, finnisch-permische usw. eingeteilt, auf alternative Baum- oder Buschmodelle oder überhaupt auf die schon traditionsreiche Debatte zur Taxonomie der uralischen Sprachen wird nicht einmal hingewiesen. Dann werden die "Methoden“ (wohl eher: methodischen Werkzeuge) der Etymologie kurz vorgestellt: vergleichende Lautgeschichte, Dialektologie, semantisches und extralinguistisch-enzyklopädisches Wissen. Am Ende der Einführung wird ferner die Strukturierung des Buches und der Wortartikel aus- führlich beschrieben. Der ganze einführende Teil ist also sehr klar und übersichtlich aufgebaut, mit Rücksicht auf das große und nicht unbedingt sprachwissenschaftlich ausgebildete Zielpublikum.

Anders als die finnischen etymologischen Wörterbücher Suomen kielen etymologinen sanakirja und Suomen sanojen alkuperä, die auch Dialekt- und sonstige der Hochsprache fremde Wörter beinhalten, und auch anders als das Nykysuomen etymologinen sanakirja von Kaisa Häkkinen, basiert das neue estnische Wörterbuch nur auf dem Erbwortschatz der Standardsprache. Genauer gesagt, es werden nur die estnischen Wortstämme behandelt, die im Wörterbuch Eesti õigekeelsussõnaraamat (ÕS) 2006 vertreten sind. Dialektwörter (wie z. B. das in der etymologischen Literatur seit jeher herumgeisternde rätselhafte viisama 'belauern', eine angebliche Entsprechung z. B. des ung. vigyáz) oder spannende Wörter der alten Schriftsprache, egal wie interessant sie aus etymologischer Sicht sein könnten, bleiben also außer Acht. So auch die Internationalismen und die jüngeren Fach- und Fremdwörter (z. B. aatom, sprinkler, raadio), Ausrufwörter und Eigennamen. Dagegen sind einige weit verbreitete umgangssprachliche junge Lehnwörter doch ins Wörterbuch aufgenommen worden, so wie 
z. B. tibla (Schimpfwort für 'Russe'). (Hier werden wahrscheinlich viele LeserInnen überrascht: Nach der Meinung der Redaktion stammt dieses Wort nicht aus russ. ty bljad' 'du Hure!' sondern aus vitebljanin 'Einwohner des Gouvernements Witebsk.)

Ausgegangen wird von Wortstämmen, und die Ableitungen, bei denen die Verbindung zum Stammwort für das heutige Sprachgefühl nicht mehr klar ist (z. B. katma 'decken' und kaas 'Deckel'), bekommen höchstens einen eigenen „Referenzeintrag“, wo mit einem Pfeil auf das Grundwort hingewiesen wird (katma [...] $\uparrow$ kaas). Auch etymologisch zusammengehörende Homonyme werden unter einem einzigen Lemma behandelt, so z. B. nõu 'Rat(schlag)' und nõ ' 'Gefäss'.

Die Wortartikel sind einfach und sehr übersichtlich strukturiert. Es werden nur ganz wenige Kürzel und Symbole verwendet. Pfeile stehen für Hinweis $(\uparrow)$ oder Herkunft $(\leftarrow)$; mit • beginnt die Aufzählung von verwandten Wörtern aus den anderen fiu. Sprachen, die Raute $\diamond$ markiert die Liste von Ableitungen, die etymologisch zum Lemmawort gehören (z. B. s. v. esi: $\diamond$ edel, edenema, edev, edu, eelis, eemal, ees, etik, etlema, ette, õdus). Die Entscheidung, immer zuerst die Formen der Lehngebersprache (evtl. mit etymologischen Entsprechungen) und erst dann die Entsprechungen in den verwandten Sprachen zu geben, ist an und für sich konsequent, kann aber etwas unlogisch wirken. Würden zuerst die fiu. Entsprechungen und erst dann die Formen der Lehngebersprache(n) gegeben, würde man implizit auch eine Vorstellung von der Chronologie der Übernahme bekommen: je älter das Lehnwort, desto mehr fiu. Entsprechungen stehen oberhalb vom Lehnoriginal.

Die etymologischen Entsprechungen in fiu. oder Lehngebersprachen sind nicht in einem Textblock platziert sondern untereinander, was zwar kein Papier spart, aber optisch viel angenehmer ist. Am Ende des Wortartikels steht noch ein Kommentarteil, wo mit klaren, vollständigen Sätzen über evtl. Unregelmäßigkeiten, Unklarheiten, Parallelfälle oder Beziehungen zu anderen Wortstämmen berichtet wird. Z. B. endet der Wortartikel kaasas 'mit', dessen Stamm (*kansa) aus dem Germanischen stammt (< urgerm. * $\chi a n s \bar{a})$, mit einem solchen Kommentarteil:

'Ist auch für einen finnischugrischen Stamm gehalten worden, dessen Entsprechungen in weiter entfernt verwandten Sprachen saamisch guos'si 'Gast' oder saamisch gázzi 'Gesellschaft; Wohngemeinschaft; Ban- 
de' (diese können auch parallele Entlehnungen aus dem Germanischen darstellen), udmurtisch kuz 'Paar' und komi goz 'Paar' sind. ka, kah ['auch'] und kas [Fragepartikel] sind durch Verkürzung aus der in der alten Schriftsprache belegten Partikel kaas entstanden. Aus demselben Wort stammt die Endung des Komitativs - ga. Wahrscheinlich aus dem Estnischen entlehnt ist livisch kas [...]' (Übers. Rez.)

Die Wörter der anderen fiu. Sprachen werden in einer vereinfachten Transkription oder in der Schriftweise der Standardsprache gegeben. Besonders zu begrüßen ist die Verwendung der nordsaamischen offiziellen Schreibweise statt der Nielsen-Orthographie. Aber warum ist z. B. im Wepsischen das "halbvokale" $i$ nicht einfach durch $j$ ersetzt worden, wenn sonst z. B. die ingrischen stimmlosen Mediae nicht mit Kapitälchen - B D G - sondern einfach mit $b d g$ geschrieben werden?

Quellenhinweise werden nicht gegeben, was etwas irritierend wirken kann, z. B. wenn auf etymologische Streitfragen hingewiesen wird. So heißt es im Kommentar zu nääl 'Schwager': „Es ist vermutet worden, dass der Stamm ursprünglich mit dem vordervokalischen Stamm ident ist, der in entfernter verwandten Sprachen 'Frau' oder 'weibliches
Tier' bedeutet ( $\uparrow$ naine), aber dies hält man für sehr zweifelhaft. "Wird sich kein/e LeserIn fragen: Wer hat vermutet, wer hält dies für zweifelhaft, und warum? Auch das vierseitige Literaturverzeichnis am Ende des Einführungsteils stellt eher eine Auswahlbibliografie dar.

Die Darstellung der allerältesten Erbwortschichten bietet keine besonderen Überraschungen. (Dies ist umso erfreulicher, da sowohl Iris Metsmägi (Audova) als auch SvenErik Soosaar früher gemeinsam mit ihrem Lehrer Ago Künnap einige Artikel über die uralische Sprachgeschichte publiziert haben, die höflich gesagt „non-mainstream“ Auffassungen vertreten.) Bei den möglichen ,interphyletischen“ Anknüpfungen wurde allerdings offensichtlich eine prinzipielle Entscheidung getroffen: Uralte Wörter, die eine mögliche Entsprechung im Indogermanischen haben, werden oft problemlos für idg. Lehnwörter gehalten, wie z. B. nimi 'Name', vedama 'ziehen', viima 'wegbringen' usw., mit Fragezeichen auch vesi 'Wasser'; beim Fragewortstamm $k u$ - dagegen wird auf andere Sprachfamilien überhaupt nicht hingewiesen. Wo aber ähnlich lautende Wörter in anderen eurasischen Sprachfamilien zu finden sind, wird nur die Ähnlichkeit erwähnt, z. B. s.v. keel 'Zunge, Sprache': 'Lautlich und bedeutungsmäßig ähnliche Stämme 
gibt es auch in Sprachen anderer Sprachfamilien [...]'.

Bei Lehnwörtern wird das Original in der Lehngebersprache, evtl. auch Reflexe oder Entsprechungen dieses Wortes gegeben - auf welcher Grundlage diese gewählt worden sind, ist nicht immer nachvollziehbar. (Warum stehen unter püha 'heilig' neben dem fraglichen germ. Original *wī $a$ - sowohl ahd. wìhen als auch ndt. weihen, während unter pühkima 'wischen' (?< urgerm. *wiskian) nur ahd. wisken gegeben wird?) Im Kommentarteil wird auf etymologisch verwandte Lehnwörter hingewiesen, $z$. B. unter hirv 'Hirsch' (< balt.) auf die frühere indoiranische Entlehnung aus demselben idg. Stamm, sarv 'Horn' (und unter sarv noch weiter auf kõrv 'Ohr'). Sehr oft werden im Kommentarteil parallele Etymologien erwähnt, oft auch ohne sie explizit in mehr oder weniger plausible einzuteilen. Oder es werden einfach mehrere mögliche Entlehnungsquellen ohne Kommentare untereinander aufgezählt, so wie bei pitsat 'Stempel' (< russ. pečat' oder $<$ dt. Petschaft). (Dass das dt. Wort ein Lehnwort aus dem Slawischen darstellt, wird nicht erwähnt. Auch nicht, dass die standardestnische Form eher aus dem Russischen, die Dialektform petsa $(h) t$ vielleicht eher aus dem Deutschen stammt.)
Besonders interessant sind natürlich die spezifisch estnischen Wortschichten: die Entlehnungen aus dem Niederdeutschen und Schwedischen, aus dem Lettischen und Russischen sowie die zahlreichen Neologismen, die aus dem Finnischen entlehnt oder - diese zweifellos sehr treffende Formulierung wird oft verwendet - „mit Unterstützung der finnischen Sprache in die Hochsprache eingeführt" wurden. Z. B. turv 'Schutz, Sicherheit' war vielleicht in estnischen Dialekten vorhanden, seine Präsenz in der Schriftsprache verdankt es trotzdem der schwesterlichen Unterstützung des Finnischen (woraus auch die Ableitung turvaline 'sicher, geschützt' entlehnt wurde). Oft wird auch die Möglichkeit, dass die in nordostestn. Küstendialekten vorhandenen Wörter eigentlich Lehnwörter aus dem Finnischen darstellen, direkt anerkannt.

Bei den späteren germanischen Lehnwörtern ist die Lehngebersprache bekanntlich nicht immer eindeutig identifizierbar, und dann werden oft mehrere Möglichkeiten angeboten: z. B. kann passima 'passen' aus dem Nieder- oder dem Hochdeutschen stammen. Die estnisch-schwedischen Dialektkontakte dagegen scheinen oft besonders ausführlich behandelt $\mathrm{zu}$ werden; im Redaktionsteam ist Meeli Sedrik 
Spezialistin dieses Gebietes. Selbstverständlich ist es nur gut, dass den sonst nicht allzu gut bekannten und nunmehr praktisch ausgestorbenen schwedenestnischen Dialekten Aufmerksamkeit gewidmet wird. Aber wie relevant ist die Frage, ob iil 'Windbö' einfach aus dem Schwedischen (il) im allgemeinen oder spezifisch aus dem Estlandschwedischen (ebenso il!) stammt?

Auf die große Debatte um Expressivwörter und Deskriptivität, die in der finnischen Etymologieforschung besonders um das SSA herum hohe Wellen geschlagen hat, wird in diesem Buch nicht näher eingegangen. Expressivwörter werden einfach häälikuliselt ajendatud 'lautlich motiviert' genannt, und die unregelmäßigen Stammvarianten, die verschiedene Konsonantenoder Vokalwechsel aufweisen, sind einfach "nahestehend“ (lähedane). Z. B. wird unter kahisema 'rauschen' auch auf die „nahestehenden Stämme" hingewiesen, die z. B. in den (semantisch ähnlichen) Verben $k i$ hama, kohama, kähisema, lahisema, nahisema, pahisema und sahisema zu sehen sind. Auch außerhalb des eigentlichen Expressivwortschatzes gibt es etymologische Verhältnisse, die hier einfach als „unregelmäßige Varianz" beschrieben werden. So, als „unregelmäßige Varianten“ voneinander, werden z. B. kirg 'Lust, Leidenschaft' und kiir 'Strahl' zusammengebündelt, ohne sie deshalb „expressiv“ zu nennen.

Mit dem neuen etymologischen Wörterbuch - dessen erste Auflage schon in einigen wenigen Wochen ausverkauft war - hat das estnischsprachige große Publikum ein klar und übersichtlich strukturiertes, sehr brauchbares Nachschlagewerk bekommen. Auch für die Linguisten, die zwar v. a. die Quellenhinweise bemängeln werden, füllt das Buch eine große Lücke - in Erwartung des künftigen „wissenschaftlichen etymologischen Wörterbuches", das auf der Website des Eesti Keele Instituut unter den Aufgaben der finnisch-ugrischen Abteilung erwähnt wird.

Johanna Laakso 